\title{
Ultrathin fibre coatings on nanofibrous nonwovens by plasma enhanced chemical vapor deposition
}

Michael Teske, Institute for Biomedical Engineering, Rostock University Medical Center, Rostock, Germany, e-mail: michael.teske@uni-rostock.de

Sabine Illner, Institute for Biomedical Engineering, Rostock University Medical Center, Rostock, Germany, e-mail: sabine.illner@uni-rostock.de

Jana Markhoff, Institute for Biomedical Engineering, Rostock University Medical Center, Rostock, Germany, e-mail: jana.markhoff@uni-rostock.de

Niels Grabow, Institute for Biomedical Engineering, Rostock University Medical Center, Rostock, Germany, e-mail: niels.grabow@uni-rostock.de

Stefan Oschatz, Institute for Biomedical Engineering, Rostock University Medical Center, Rostock, Germany, e-mail: stefan.oschatz@uni-rostock.de

\section{Introduction}

To improve the implant-tissue interaction of medical devices often specific coatings are used, considering the requirements of the implant and the surrounding tissue. Plasma enhanced chemical vapor deposition (PECVD) is a method for the generation of ultrathin tailored polymer coatings, especially due to the diversity of usable monomers and process conditions. Moreover, even complex geometries can easily be coated. The feasibility of different PECVD coatings on nanofibrous nonwovens, which is currently rarely described in the literature, is investigated in this study. Also the possibility to modulate the biological response by varying the PECVD process was examined in vitro.

\section{Methods}

PECVD was carried out with the monomers hexamethyldisiloxane (HMDSO) and allylamine on a silicone-based polycabonaturethane (TSPCU) nanofibrous nonwoven as substrate. The nonwovens were morphological characterized via SEM and direct contact tests with endothelial cells and fibroblasts on coated and untreated nonwovens were performed. Moreover, plasma polymers were verified by EDX and ATR-IR measurements.

\section{Results}

Depending on the plasma process parameters fibre thickness measurements revealed mostly only minor changes in nanometer range in comparison to the untreated TSPCU fibres. ATR-IR spectroscopy also confirmed the ultrathin layers. The EDX measurements showed an increase of nitrogen in allylamine coatings and silicon in HMDSO coatings. Influence of PECVD coatings on the relative cell viability in comparison to the untreated TSPCU nonwoven was observable.

\section{Conclusion}

The morphological analyses revealed the feasibility of plasma coatings on nanofibrous nonwovens without structural changes or film formation. Furthermore, chemical analyses confirmed the generation of plasma polymer coatings. The biological response of cells by the PECVD process is often reported in literature. 


\section{Method to determine particle release during long-term loading for as- sessment of coating durability of cardiovascular stents}

Wolfram Schmidt, Rostock University Medical Center, Institute for Biomedical Engineering, Rostock, Germany, wolfram.schmidt@uni-rostock.de

Christoph Brandt-Wunderlich, Institute for ImplantTechnology and Biomaterials IIB e.V., Rostock, Germany, christoph.brandt@iib-ev.de

Anja Kurzhals, Rostock University Medical Center, Institute for Biomedical Engineering, Rostock, Germany, anja.kurzhals@uni-rostock.de

Klaus-Peter Schmitz, Institute for ImplantTechnology and Biomaterials IIB e.V., Rostock, Germany, and Rostock University Medical Center, Institute for Biomedical Engineering, Rostock, Germany, schmitz@iib-ev.de

Niels Grabow, Rostock University Medical Center, Institute for Biomedical Engineering, Rostock, Germany, niels.grabow@uni-rostock.de

\section{Introduction}

Many catheters and vascular implants are coated to increase biocompatibility or to reduce friction during catheter based implantation. Several regulations require assessment of coating durability over the implants life time. An in vitro method for stent testing is presented to measure released particulate matter at defined inspection intervals.

\section{Method}

The stents are implanted in clean test tubes filled with particle free test solution (Ampuwa, Fresenius Kabi). The tubes are sealed to avoid contamination. Then, the fatigue test is started. Intermediate and final inspections are performed by stopping the loading, flushing the test tubes and collecting test solution for particulate evaluation. A test setup for fatigue analysis of coronary stents in a bent configuration (tube ID=5 mm) was investigated exemplarily. Polystyrene microspheres with a size of 10, 25 and $50 \mu \mathrm{m}$ (COUNT-CAL тм Count Precision Size Standards, Thermo Scientific) were used to check for particle recovery $(n=6)$. Two cleaning steps followed. Particle counting was performed by light obscuration method (HIAC 9703, sensor HRLD-400, Beckman Coulter).

\section{Results}

Initially, the test tubes contained $\leq 3$ particles $/ \mathrm{ml}$. The recovery rate was $103 \pm 5 \%(10 \mu \mathrm{m}), 94 \pm 4 \%(25 \mu \mathrm{m})$ and $78 \pm 12 \%$ $(50 \mu \mathrm{m})$, respectively. Less than $3 \%$ of the particles were found in the cleaning solutions.

\section{Conclusion}

The recovery rate meets the requirements of FDA guidances ( $\geq 90 \%$ for $10 \mu \mathrm{m}$ and $25 \mu \mathrm{m}$ particles, $\geq 75 \%$ for $50 \mu \mathrm{m}$ particles). As the cleaning solutions showed only a small number of particles, it is assumed that the lower recovery rate for the $50 \mu \mathrm{m}$ particles is due to sinking effects. Our method using a fixed volume during stent loading can be adapted to all durability testers where tubes are used to fix the stents (radial pulsatile, bending, axial compression, torsion).

The test solutions are available for further characterization of particulates by dynamic imaging analysis, scanning electron microscopy or Raman spectroscopy, respectively. 


\section{Scaffolds with magnetic nanoparticles for tissue stimulation}

Sara Leal-Marin, Institute for Multiphase Processes, Leibniz University Hannover, Garbsen, Germany, Lower Saxony Center for Biomedical Engineering, Implant Research and Development, Hannover, Germany, lealmarin@imp.uni-hannover.de

Glynn Gallaway, Institute for Multiphase Processes, Leibniz University Hannover, Garbsen, Germany, School of Mechanical Engineering, Purdue University, Indiana, USA, ggallawa@purdue.edu

Kai Höltje, Institute for Multiphase Processes, Leibniz University Hannover, Garbsen, Germany, hoeltje@imp.uni-hannover.de

Alex Lopera-Sepulveda, Grupo GICEI-GIIEN, Institución Universitaria Pascual Bravo,Medellin, Colombia, Grupo de Cerámicos y Vítreos, Escuela de Física, Universidad Nacional de Colombia, Medellín, Colombia, a.lopera1420@pascualbravo.edu.co

Birgit Glasmacher, Institute for Multiphase Processes, Leibniz University Hannover, Garbsen, Germany, Lower Saxony Center for Biomedical Engineering, Implant Research and Development, Hannover, Germany, sekretariat@imp.uni-hannover.de

Oleksandr Gryshkov, Institute for Multiphase Processes, Leibniz University Hannover, Garbsen, Germany. Lower Saxony Center for Biomedical Engineering, Implant Research and Development, Hannover, Germany, gryshkov@imp.unihannover.de

\section{Introduction}

Magnetic nanoparticles (MNPs) have widely been employed in medicine in targeted hyperthermia therapies, as markers for resonance tomography, sensors for diagnostic and localized drug delivery systems. A combination of MNPs with a biocompatible scaffold could be used to improve nerve and muscle regeneration through directed stimulation of cellular activities via application of external magnetic fields. In this study we aimed to compare structural properties and biocompatibility of polymeric scaffolds with magnetite and cobalt ferrite nanoparticles.

\section{Methods}

Solid films (dip coating) and scaffolds (electrospinning) were obtained using 14\% (w/v) polycaprolactone (PCL) with different MNP-concentrations $(5 \%, 7.5 \%(\mathrm{w} / \mathrm{v}))$ of magnetite $\left(\mathrm{Fe}_{2} \mathrm{O}_{3}\right)$ or cobalt ferrite $\left(\mathrm{CoFe}_{2} \mathrm{O}_{4}\right)$. MNPs were dispersed in the PCL solution (in 2,2,2-Trifluoroethanol) using an ultrasonic bath. The morphological properties (scanning electron microscopy, SEM), chemical composition (Raman spectroscopy), wettability (contact angle) and magnetic behaviour (magnet attraction) were evaluated. The biocompatibility was assessed using human bone marrow mesenchymal stem cells seeded on the UV-sterilized scaffolds $\left(\varnothing 12 \mathrm{~mm}, 25.000\right.$ cells $\left./ \mathrm{cm}^{2}\right)$. The cell-scaffold interactions were analysed using SEM. The cell viability and metabolic activity was evaluated by a the Calcein-AM/Ethidium homodimer-1 and Resazurin reduction assays over 10 days, respectively.

\section{Results}

The obtained films and scaffolds with MNPs presented noticeable magnetic behaviour, which increased with an increase in MNPs concentration, and was higher for cobalt ferrite. The solid films presented a smooth surface with some agglomerated MNPs. The electrospun scaffolds presented an average fiber diameter of $1.8 \pm 0.4 \mu \mathrm{m}$ with few MNP agglomerations. Raman spectroscopy identified the main band characteristic to the MNPs and PCL. Both films and scaffolds allowed the cells to attach and be metabolically active.

\section{Conclusion}

The obtained scaffolds presented a good dispersion of the MNPs showing magnetic behaviour and high biocompatibility for TE applications. Further work will focus towards analysing the mechanical properties and development of a radiofrequency-based stimulation system.

Acknowledgements This work was supported by the German Academic Exchange Service (DAAD, project number 91725466), Caroline Herschel Program Leibniz University Hannover and Fulbright Germany U.S. Student Research Grant. 


\section{Stability and Biocompatibility Tests of Alginate as Cochlear Implant Coating Material}

Claas-Tido Peck, Department of Otolaryngology, Hannover Medical School, Hanover, Germany, Cluster of Excellence "Hearing4all”, Hannover, Germany, Peck.Claas-Tido@mh-hannover.de Jana Schwieger, Department of Otolaryngology, Hannover Medical School, Hanover, Germany, Cluster of Excellence "Hearing4all”, Hannover, Germany, Schwieger.Jana@mh-hannover.de Thomas Lenarz, Department of Otolaryngology, Hannover Medical School, Hanover, Germany, Cluster of Excellence "Hearing4all”, Hannover, Germany, Lenarz.Thomas@mh-hannover.de Verena Scheper, Department of Otolaryngology, Hannover Medical School, Hanover, Germany, Cluster of Excellence "Hearing4all”, Hannover, Germany, Scheper.Verena@mh-hannover.de

\section{Introduction}

Inner ear trauma caused by cochlear implantation (CI) is a severe clinical problem. It was shown that an electrode alginate coating can reduce the insertion forces in vitro. The grade of viscosity can be adjusted by using different metal ions for crossliniking the salts of the alginic acid. The aim was to investigate the stability and inner ear biocompatibility of alginate crosslinked with either $\mathrm{CaCl}_{2}$ or $\mathrm{BaCl}_{2}$.

\section{Methods}

Alginate beads were crosslinked with either $\mathrm{CaCl}_{2}$ or $\mathrm{BaCl}_{2}$. The beads were cultivated in artificial perilymph and stability and swelling were observerd over 6 months. Ototoxicity was tested on cochlea whole mount explants from neonatal rats. Neomycin served as positive control to induce hair cell damage and explants without any addition served as negative control. After 48 hours the hair cell survival was analysed microscopically.

\section{Results}

Neomycin treatment induced an extensive inner and outer hair cell loss. Neither $\mathrm{CaCl}_{2}$ nor $\mathrm{BaCl}_{2}$ crosslinked alginate beads caused any damage to the hair cells. Even though the same volume of alginate and crosslinkers were used, $\mathrm{CaCl}_{2}$ crosslinked beads were initially almost double the size of $\mathrm{BaCl}_{2}$ crosslinked beads. None of the crosslinked alginate beads had a significant volume change within 3 months being cultured in artificial perilymph. After 3 months $\mathrm{CaCl}_{2} \mathrm{Crosslinked}$ beads swelled significantly and dissolved within one week whereas $\mathrm{BaCl}_{2}$ crosslinked alginate beads were unchanged until 6 months after culture start.

\section{Conclusion}

Both crosslinkers are compatible with the inner ear sensory epithelium and ensure a stable gelation of alginate with significant swelling of the $\mathrm{CaCl}_{2}$ crosslinked beads after 3 months. For coatings which need to be long term stable $\mathrm{BaCl}_{2}$ should be chosen whereas $\mathrm{CaCl}_{2}$ may be more suitable for applications where limited stability is needed. 


\section{Neural electrode impedance reduction via Pulsed-DC electrophoretic dep- osition}

Vaijayanthi Ramesh, Institute of Technical Chemistry I, University of Duisburg-Essen, Essen, Germany, vaijayanthi.ramesh@uni-due.de

Christoph Rehbock, Institute of Technical Chemistry I, University of Duisburg-Essen, Essen, Germany, christoph.rehbock@uni-due.de

Svilen D. Angelov, Department of Neurosurgery, Hannover Medical School, Hannover, Germany, Angelov.Svilen@mh$\underline{\text { hannover.de }}$

Kerstin Schwabe, Department of Neurosurgery, Hannover Medical School, Hannover, Germany, Schwabe.Kerstin@mh$\underline{\text { hannover.de }}$

Joachim K Krauss, Department of Neurosurgery, Hannover Medical School, Hannover, Germany, Krauss.Joachim@mhhannover.de

Stephan Barcikowski, Institute of Technical Chemistry I, University of Duisburg-Essen, Essen, Germany, stephan.barcikowski@uni-due.de

\section{Introduction}

Later stages of Parkinson's disease induce severe tremors in elderly patients seriously impairing their daily life, particularly when drug-based treatments have ceased to work. To improve the patients' life quality, deep brain stimulation (DBS) using implanted platinum neural electrodes are performed, providing electric pulses to subthalamic nucleus (STN) and in turn tune neuronal activity. Even though this treatment is routinely applied in clinical practice, difficulties such as low stimulation efficiency and reduced battery capacity of the pulse generator due to impedance increase occurs over time.

\section{Methods}

Coating of 3D-electrode surfaces is done using electrophoretic deposition (EPD) in a custom-designed chamber with a field strength of $5 \mathrm{~V} / \mathrm{cm}$ and a platinum mass concentration of $100 \mu \mathrm{g} / \mathrm{ml}$. We compared the impact of DC (5 min) and pulsed-DC EPD (period of $1 \mu \mathrm{s}$ and a duty cycle of $50 \%, 10 \mathrm{~min}$ ) on stimulation conditions.

\section{Results}

We found that the pulsed-DC coatings yielded a more homogeneous surface coverage and also the impedance is significantly reduced in contrast to the uncoated controls, an effect which could not be observed for DC-EPD.

\section{Conclusion}

In consecutive studies, the coated electrodes will undergo long term in vitro stimulations in saline solutions and in vivo stimulations in rat models and the influence of DC and pulsed-DC fields on impedance will be evaluated. 


\section{Kill\&Repel: Antimicrobial and antifouling coating for wound dressings based on biohybrids}

Manuela Garay-Sarmiento, DWI - Leibniz Institute for Interactive Materials e.V, RWTH Aachen University, Aachen, Germany, garay@dwi.rwth-aachen.de

Mariia. Vorobii, DWI - Leibniz Institute for Interactive Materials e.V, RWTH Aachen University, Aachen, Germany, vorobii@dwi.rwth-aachen.de

Lena Witzdam, DWI - Leibniz Institute for Interactive Materials e.V, RWTH Aachen University, Aachen, Germany, witzdam@dwi.rwth-aachen.de

Niklas Herrmann, DWI - Leibniz Institute for Interactive Materials e.V, RWTH Aachen University, Aachen, Germany, nherrrmann@dwi.rwth-aachen.de

Christian Simons, DWI - Leibniz Institute for Interactive Materials e.V, RWTH Aachen University, Aachen, Germany, simons@dwi.rwth-aachen.de

Dr. A. de los Santos Pereira, Institute of Macromolecular Chemistry, Prague, Czech Republic, santospereira@imc.cas.cz

Dr. E. Heine DWI - Leibniz Institute for Interactive Materials e.V, RWTH Aachen University, Aachen, Germany, heine@dwi.rwth-aachen.de

Prof. R. Lütticken, DWI - Leibniz Institute for Interactive Materials e.V., RWTH Aachen University, Aachen, Germany, rodriguez@dwi.rwth-aachen.de

Dr. F. Jakob, DWI - Leibniz Institute for Interactive Materials e.V., RWTH Aachen University, Aachen, Germany, rodriguez@dwi.rwth-aachen.de

Prof. U. Schwaneberg, DWI - Leibniz Institute for Interactive Materials e.V., RWTH Aachen University, Aachen, Germany,rodriguez@dwi.rwth-aachen.de

Cesar Rodriguez-Emmenegger, DWI - Leibniz Institute for Interactive Materials e.V., RWTH Aachen University, Aachen, Germany, rodriguez@dwi.rwth-aachen.de

\section{Introduction}

Even the most advanced antimicrobial dressings cannot fully protect and treat infected wounds because bacteria and their debris can adhere to the dressing, blocking the active antimicrobial surface. Moreover, dermal cells can attach causing adherence of the dressing to the wound and laceration by dressing exchange. Herein, we propose an ultra-thin Kill\&Repel coating that simultaneously kills bacteria and repels adhesion of their debris and dermal cells.

\section{Methods}

Surface activity of the liquid chromatography peak I peptide (LCI) is exploited to achieve irreversible binding of ProteinPolymer (LCI-Pol) and Protein-Enzyme (LCI-Endlys) hybrids to the surface of dressings via physical interactions. Antifouling properties were introduced by grafting carboxybetaine-methacrylamide and N-hydroxypropylmethacrylamide chains directly from the LCI. The adsorption of LCI on the generated a brush-like coating. Bactericidal activity was achieved by fusing the LCI with endolysin, which cleaves the bacteria's wall at specific sites. Different wound dressings were functionalized by incubation in a mixture of LCI-Pol and LCI-Endlys.

\section{Results}

The assembly of hybrids on surfaces was studied by SPR and followed a Langmuir isotherm. X-Ray reflectivity showed two distinguishable layers on the surface corresponding to the AMP and polymer chains, thus indicating surface-oriented adsorption of the LCI peptide. The Kill\&Repel modification prohibited the adsorption of proteins, the adhesion of dermal cells as well as colonization of the dressing even after $24 \mathrm{~h}$ contact with bacteria. LCI-Endlys was able to kill bacteria on direct contact. The inhibition of bacterial growth was limited to the area under the modified dressing, confirming a nonleachable coating system. Furthermore, the coating did not show any harming effect on eukaryotic cells resulting in a non-cytotoxic but effective strategy.

\section{Conclusion}

The Kill\&Repel coating was transfered to different dressings resulting in a drastic increase in performance. Such excellent performance is particularly remarkable considering that the coating is a one-molecule-thick coating. 